\title{
Partial delivery: Opportunity or threat for wine cooperatives
}

\author{
J.H. Hanf, L. Bitsch, and U. Schilling \\ Hochschule Geisenheim University, von-Lade-Str. 1, 65366 Geisenheim, Germany
}

\begin{abstract}
Numerous wine cooperatives exclude partial delivery by a strict and unexceptional full delivery obligation. However, in practice, some well-known representatives of the German wine cooperative system, such as Moselland eG, successfully follow the partial delivery procedure. In addition, cooperative members use various measures in practice to avoid the full delivery obligation, e.g. through the legal division or splitting of the member companies. Nevertheless, the procedure is currently barely investigated and researched, and since neither the DRV nor the auditing cooperative associations carry out surveys on the partial delivery, it is not possible to determine the exact significance of this for the German winegrowers' cooperative system (Jung, 2016). Due to the current situation with regard to partial delivery in the German wine cooperative system, the aim of this paper is to present the concept of partial delivery and to discuss challenges for the cooperative system.
\end{abstract}

\section{Einleitung}

Die Genossenschaften der Weinbranche unterscheiden sich nicht nur hinsichtlich ihrer Vermarktungskanälen oder hinsichtlich der Größe der erfassten Rebflächen, sondern sie verfolgen auch verschiedene Verfahren der Traubenanlieferung, welche die Voll- oder die Teilablieferung der produzierten Trauben darstellen. Die Teilablieferung ist realisierbar, da es Mitgliedern mancher Genossenschaften durch die im Genossenschaftsgesetz niedergeschriebenen Ablieferungsrichtlinien nicht verboten ist, ihre Trauben auch zu besseren Konditionen an andere Marktteilnehmer zu verkaufen (Frick, 2004).

Die Mehrheit der Winzergenossenschaften (WGs) schließen dieses Vorgehen jedoch durch eine strikte und ausnahmslose Vollablieferungsverpflichtung aus und auch der Deutsche Raiffeisenverband (DRV) und die zuständigen regionalen Genossenschaftsverbände sehen dieses Vorgehen als eher negativ belastet an (Hein, 2016). Erfahrungen aus Österreich prägen das negative Bild, da dort WGs, durch das offene und wenig kontrollierbare Verfahren der Teilablieferung wirtschaftliche Probleme bekamen. Dabei spielten vor allem die schwankenden Erntemengen der Teilablieferer eine entscheidende Rolle. In ertragsarmen Jahren haben sie die angelieferten Mengen erheblich reduziert, um ihren zweiten Vermarktungsweg ausreichend bedienen zu können (Glatt, 2016). Demnach blieben nur kleinere Mengen, meist schlechterer Qualität, für die Genossenschaften übrig. Es gibt jedoch keine Hinweise darauf, dass eine WG wirtschaftlich ausschließlich aufgrund der Teilablieferung gescheitert ist.

Trotz dieser Erfahrungen verfolgen einige bekannte Vertreter des deutschen Winzergenossenschaftswesens, wie z.B. die Moselland eG, das Verfahren der Teilablieferung erfolgreich. Zusätzlich nutzen Genossenschaftsmitglieder in der Praxis verschiedene Maßnahmen die Vollablieferungspflicht zu umgehen. Bspw. ergreifen
Mitglieder Maßnahmen wie die legale Teilung bzw. Splittung der Mitgliedsbetriebe. Der Betrieb mit der ursprünglichen Betriebsnummer bleibt vollablieferndes Mitglied einer WG; jedoch mit weniger eingebrachten Rebflächen. Der neu gegründete Betrieb kann mit den restlichen Flächen völlig frei einer alternativen Vermarktung nachgehen.

Aufgrund der aktuellen Situation hinsichtlich der Teilablieferung im deutschen Winzergenossenschaftswesen ist es Ziel dieser Arbeit das Konzept der offiziellen und "inoffiziellen" Teilablieferung darzustellen und Herausforderungen für das Genossenschaftswesen zu diskutieren.

Obwohl die Teilablieferung in der Praxis Anwendung findet, ist es zurzeit nur wenig untersucht und erforscht. Da weder der DRV, noch die prüfenden Genossenschaftsverbände Erhebungen zur Teilablieferung durchführen, kann eine genaue Bedeutung dieser für das deutsche Genossenschaftswesen nicht erschlossen werden (Jung, 2016). Die Teilablieferung in WGs ist bisher in der Literatur nicht explizit behandelt. Auch die Ausweitung der Recherche auf das Verfahren der Teilablieferung im gesamten Genossenschaftswesen blieb erfolglos, weshalb zur Erfassung des Verfahrens auf Expertenwissen zurückgegriffen ${ }^{1}$. Es wurden Geschäftsführer und Vorstandsvorsitzende von WGs befragt, die Teilablieferung in ihrem Unternehmen zulassen. Des Weiteren wurden Mitarbeiter verschiedener regionaler Genossenschaftsverbände befragt, da sie durch die Prüfungen der jeweiligen WGs ebenfalls mit dem Verfahren vertraut sind. Um Teilablieferung besser beschreiben und begreifen zu können, wird es im Folgenden dargestellt. Weiterhin werden die durch die Theorie gestützten Herausforderungen für Genossenschaften erarbeitet. Die empirische Erhebung dieser Arbeit stützt sich auf den explorativen Ansatz der Marktforschung von Fantapié Altobelli (2007). Es wurden 20 Experteninterviews geführt, welche mit einer qualitativen Inhaltsanalyse nach Gläser \& Laudel (2010) ausgewertet wurden. 


\section{Teilablieferung in deutschen Winzergenossenschaften}

Die Satzungen der WGs sehen generell eine Vollablieferungspflicht als Voraussetzung an. Lediglich in Ausnahmefällen ist es erlaubt, nur Teilflächen an die WG zu liefern. Diese Ausnahmen bedürfen einer Zustimmung des Vorstands (Heßdörfer, 2016). Praktisch bedeutet dies, dass prinzipiell jedes Mitglied einer WG Teilablieferer werden kann, sofern die WG dieses Verfahren grundsätzlich zulässt. Teilablieferer verfolgen, neben der Mitgliedschaft in der WG noch weitere Vermarktungswege, wie z. B. einen Trauben-, Fass- oder Flaschenweinverkauf. Die übrigen Flächen des Mitgliedsbetriebes können somit selbstständig und ohne Vorgaben auf dem freien Markt vermarktet werden. Die Mustersatzung des DRV verbietet ihnen durch $\S 12$ die Trauben ihrer übrigen Rebflächen an eine andere WG, eine Erzeugergemeinschaft oder an ein ähnliches Unternehmen zu liefern (DRV, 2014: §12). Diese Bestimmung schließt aus, dass Teilflächen der Teilablieferer anderweitig in genossenschaftliche Verarbeitung gelangen und stärkt somit die Konkurrenzposition von alternativen Abnehmern, wie z.B. Weingüter oder Kellereien (Hanf \& Härle, 2015). Unabhängig vom Ablieferungsverfahren müssen die Mitglieder anteilig ihrer eingebrachten Rebflächen Geschäftsanteile der WG zeichnen und im Falle eines Austritts auch identische Kündigungsfristen einhalten (Heßdörfer 2016; Kießling 2016; Schilling, 2016). Die Vermarktung der eingebrachten Flächen liegt bei der WG; das teilabliefernde Mitglied darf die Trauben von diesen Flächen nicht selbstständig vermarkten. Auch auf die Stimmanteile nimmt die Teilablieferung keinerlei Einfluss. Diese werden unabhängig vom Anlieferungsverfahren verteilt, so dass jedes Mitglied genau eine Stimme erhält.

Die Auszahlungspreise je Kilogramm Trauben sind für beide Verfahren identisch. Unabhängig vom Verfahren werden zunächst Abschlagszahlungen je nach angelieferter Traubenmenge ausgezahlt. Die endgültige geldliche Vergütung der Trauben erfolgt, sobald alle Weine verkauft sind und richtet sich nach den erlösten Preisen auf dem Markt abzüglich aller entstandenen Aufwendungen (Engels, 2002). Die Ablieferungsverfahren unterscheiden sich jedoch in der Ermittlung der vermarktungsfähigen Menge (Heßdörfer 2016; Kießling 2016; Schilling 2016). Dies beruht darauf, dass zur Bestimmung des vermarktungsfähigen Kontingents unterschiedliche Ansätze verwendet werden. Bei Teilablieferern wird eine festgesetzte Menge von $13.462 \mathrm{~kg}$ Trauben je Hektar Rebfläche angenommen. Bei einer fixen Auspressquote von $78 \%$ ergibt das den Hektarhöchstertrag von 10.500 Liter Most. Sollte die tatsächliche Auspressquote unter $78 \%$ liegen, so kann es sein, dass ein Teilablieferer hierdurch nicht sein gesamtes, potentiell zur Verfügung stehendes Kontingent ausschöpfen kann und somit einen Nachteil erhält (Schilling, 2016). Der Vollablieferer hingegen wird stets nach der tatsächlich erreichten Auspressquote abgerechnet. Die WG kann also in Jahren, in denen eine niedrige tatsächliche Auspressquote $\mathrm{zu}$ erwarten ist, die zugelassenen Anlieferungsmengen pro Vollablieferer erhöhen und somit sicherstellen, dass das

\footnotetext{
${ }^{1}$ Für die Überprüfung der Annahmen bezüglich der Herausforderungen für Genossenschaften wurden die Erfahrungen dieser Experten auch einbezogen.
}

vermarktungsfähige Kontingent je Hektar Rebfläche stets erreicht werden kann.

\subsection{Ablieferungsverpflichtung und Kontrollierbarkeit}

Vollabliefernde Betriebe sind gemäß der Satzung einer WG dazu verpflichtet, ausnahmslos alle Trauben, die sie auf ihren Rebflächen ernten, an die WG abzuliefern und dürfen folglich keine Trauben anderweitig vertreiben oder verarbeiten (Schilling, 2016). Teilablieferer hingegen sind nur mit Teilen ihrer betrieblichen Rebfläche Mitglied in der WG. Für diese Teilflächen besteht eine Vollablieferungspflicht. Offiziell wird daher von "Vollablieferern von Teilflächen" gesprochen (Heßdörfer, 2016). Die Teilflächen werden zu Beginn der Mitgliedschaft exakt mittels der Kataster- und Flurstücknummer erfasst, so dass diese zumindest theoretisch nicht durch das Mitglied austauschbar sind (Schilling, 2016). Eine ausreichend, lückenlose und zweifelsfreie Rückverfolgbarkeit ist derzeit jedoch nicht gegeben (Kießling, 2016), da die technischen Mittel dies nicht ermöglichen und eine persönliche Kontrolle der Flächen zu aufwändig und zu kostspielig wäre (Heßdörfer, 2016). Es besteht die Gefahr, dass Mitglieder diese Chance nutzen und Trauben von anderen Flächen, die sie nicht in die WG eingebracht haben, anliefern. Hierdurch wird ein Austausch von unterschiedlichen Traubenqualitäten ermöglicht. Dieses Verhalten führt zu adverser Selektion seitens der Mitglieder, die die Abnahmeverpflichtung der WGs ausnutzen, um qualitativ schlechte Trauben, die andere Abnehmer abstoßen würden, zu vermarkten (Hanf et al., 2012). Hierdurch kann eine u. U. schon bestehende Qualitätsproblematik bei WGs noch verschärft werden. Des Weiteren ist es denkbar, dass durch die fehlende Kontrolle der Teilflächen die Mengenschwankungen innerhalb der WG zunehmen können. Teilablieferer haben die Möglichkeit, der Genossenschaft u. U. Trauben vorzuenthalten und diese anderweitig $\mathrm{zu}$ vermarkten oder $\mathrm{zu}$ verarbeiten. Ebenso könnten sie mehr als den Aufwuchs der gemeldeten Flächen abliefern, wodurch die Planungsunsicherheit für die Vermarktungsmenge in der Winzergenossenschaft im Zeitablauf wächst.

Annahme 1: Teilablieferer können u. U. Rebflächen innerhalb ihres Betriebes austauschen, um Vorteile für den eigenen Betrieb zu schaffen. Dies kann sich negativ auf die Qualitätsproblematik, aber auch auf Schwankungen der angelieferten Traubenmengen auswirken.

\subsection{Ziele der Mitgliedschaft}

Hinsichtlich der Ziele der Mitgliedschaft in einer Genossenschaft können Unterschiede auftauchen. Zum einen unterscheiden sich die Quellen der Betriebseinnahmen der beiden Verfahren deutlich voneinander. Während für Vollablieferer die WG die einzige Einnahmequelle darstellt, generieren Teilablieferer ihr Haupteinkommen meist aus anderen Vermarktungswegen, wie z. B. Selbstvermarktung von Weinen (Heßdörfer 2016; Kießling, 2016). Dies verdeutlicht, dass die Auszahlungen der WGs für Vollablieferer einen deutlich höheren Stellenwert einnehmen, da sie komplett von der WG abhängig sind. Trotz der Abhängigkeit haben Vollablieferer ein geringes unternehmerisches Risiko, da die Genossenschaft eine 
Abnahmeverpflichtung der Trauben hat. Teilablieferer hingegen haben durch mehrere Standbeine ihr finanzielles Risiko gestreut und sind nicht ausschließlich auf die Leistungen der WG angewiesen. Es ist zu erwarten, dass beide Seiten möglichst hohe Auszahlungen an Traubengeld verfolgen. Teilablieferer schätzen neben den Auszahlungen oft weitere Vorteile einer Mitgliedschaft, wie z. B. die sichere Abnahmegarantie von Traubenmengen und -rebsorten, die sie anderweitig nicht vermarkten können oder die Flexibilität in Form von einem erleichterten Betriebswachstum, in Bezug auf Rebfläche ohne hierfür weitere Investitionen in die eigene Betriebsstätte für bspw. eine größere Weinbereitung tätigen zu müssen.

Annahme 2: Teilablieferer sehen die Ziele einer Mitgliedschaft in einer Winzergenossenschaft nicht nur in den Auszahlungsleistungen, sondern auch in weiteren Faktoren, wie z. B. der Flexibilität in ihrer eigenen Betriebsausrichtung und im Flächenwachstum.

\subsection{Genossenschaftsverständnis}

In der Theorie gibt es zwei verschiedene Modelle für die Sichtweisen einer Genossenschaft. ${ }^{2}$ Werden diese auf die Besonderheiten und die Funktion einer WG übertragen, so ergeben sich neue, abweichende Charakteristika der jeweiligen Sichtweise. Dem grundlegenden Modell des "eigenständigen Unternehmens" nach Helmberger \& Hoos (1962:, 275) zufolge muss die WG als ein selbstständiges Unternehmen gesehen werden, das als Ziel u. a. die Weinproduktion und Vermarktung verfolgt. Die Weine werden aus den angelieferten Trauben erzeugt, die die Mitglieder auf ihren Rebflächen anbauen und vermarktet. Folglich sind WGs an Trauben interessiert, die eine möglichst hohe Qualität aufweisen und eine gute Basis dafür liefern, mit möglichst geringen Aufwendungen den Marktanforderungen entsprechende Weine zu produzieren und zu vermarkten. Die Mitglieder werden als Lieferanten gesehen und sollen, wenn möglich, die vom Kunden / Markt stark nachgefragte Rebsorten anbauen, um die Vermarktung, den Aufbau einer Marktposition und reputation einfacher zu gestalten. Die Problematik dieser Sichtweise zeigt sich jedoch, sobald die Interessen der Mitglieder betrachtet werden, die von einer (Winzer-) Genossenschaft laut Geng (2006: $\S 1$, Absatz 1) möglichst optimal gefördert werden sollen. Mitglieder identifizieren sich folglich nur wenig mit dem "eigenständigen Unternehmen" Winzergenossenschaft, weil für sie eine betriebswirtschaftlich erfolgreiche Produktion von Trauben und die Führung des eigenen Mitgliedbetriebes im Vordergrund stehen. Es ist dennoch davon auszugehen, dass Vollablieferer sich mehr mit der Genossenschaft als "eigenständiges Unternehmen" identifizieren als Teilablieferer. Vollablieferer sind gänzlich an die WG gebunden, eine Ausrichtung an deren Forderungen in Bezug auf Rebsorten, Qualitäten und Mengen ist deshalb wahrscheinlicher als bei teilabliefernden Betrieben. Bei Erfüllung der Forderungen kommt es meist zu einer gesteigerten Auszahlung und somit zu einer verbesserten Förderung der eigenen Mitgliedswirtschaft. Für Teilablieferer liegt das Hauptaugenmerk i. d. R. auf der

\footnotetext{
2 Die beiden Modelle sind ausreichend diskutiert und können bei Bedarf bei den Autoren Helmberger \& Hoos (1962) sowie bei Phillips (1953) nachgelesen werden.
}

eigenen Mitgliedswirtschaft bzw. den alternativen Vermarktungswegen, weshalb das Interesse an der WG/dem "eigenständigen Unternehmen" kleiner ausfallen kann (vgl. Heßdörfer 2016; vgl. Kießling 2016).

Die Übertragung der Sichtweise von PHILLIPS (1953) auf WGs als "der verlängerte Arm der Mitgliedsbetriebe" verdeutlicht die kontroversen Annahmen der beiden Modelle. Hierbei stehen die Interessen der Mitglieder und die bestmögliche Förderung der Mitgliedswirtschaften im Vordergrund und die Genossenschaft hat als Unternehmen keine Entscheidungskompetenz. Für die WGs bedeutet dies, dass sie möglichst hohe Auszahlungen je Hektar Rebfläche leisten sollen und es den Mitgliedern möglich sein sollte, einen einfachen Weinbau mit wenigen Aufwendungen in der Traubenproduktion verfolgen zu können. Dies ist für beide - voll- und teilabliefernde Betriebe ein wichtiges Kriterium dieser Sichtweise. Teilablieferer sehen die Optimierung ihrer eigenen Mitgliedswirtschaft meistens noch differenzierter. Neben der Höhe der Auszahlung ist für sie die Risikostreuung durch mehrere Betriebszweige, die sichere Abnahme von Trauben und die Flexibilität in der Anlieferung von Bedeutung (vgl. Zenzen 1984: 54 f). Durch die sichere Mengenabnahme erhalten sie auch eine finanzielle Sicherheit und können somit ihre Verhandlungsposition gegenüber Weinkommissionären oder Einkäufern von Kellereien im eigenen Betrieb stärken. Sie müssen Weine nicht $\mathrm{zu}$ allen Preisen verkaufen, sondern sie können durch die Teilmitgliedschaft in der WG risikoärmer spekulieren und somit agiler verhandeln. Die Mitgliedschaft in der WG ist für Teilablieferer folglich ein Teil ihrer persönlichen strategischen Betriebsausrichtung und spiegelt eine stärkere Identifikation mit der Sichtweise Phillips wider. Durch die verschiedenen Interessen und unterschiedlichen Vorgehen der einzelnen Mitgliedswirtschaften zur Zielerreichung können Probleme und Konflikte innerhalb der Winzergenossenschaft hervorgerufen werden (Bijmann, 2005).

Annahme 3: Die strategischen Betriebsausrichtungen von Voll- und Teilablieferern unterscheiden sich. Teilablieferer legen den Fokus auf ihren eigenen Betrieb, während Vollablieferer sich stärker auf die Winzergenossenschaft konzentrieren.

\section{Methodik der qualitativen Erhebung}

Die empirische Erhebung dieser Arbeit stützt sich auf den explorativen Ansatz der Marktforschung von Fantapié Altobelli (2007). Die Methodik findet vorwiegend Anwendung bei neuartigen und komplexeren, oder bisher schlecht strukturierten Forschungsgebieten und steht somit den Methoden der deskriptiven und kausalen Studien gegenüber, die sich in der Regel mehr mit bereits bekannten Problemstellungen, deren Häufigkeiten im Auftreten, Entwicklungen oder Ursache-Wirkungs- Beziehungen auseinandersetzen (Fantapié Altobelli, 2007). Es wurden insgesamt 20 Experteninterviews geführt und nach der qualitativen Inhaltsanalyse von Gläser \& Laudel (2010) ausgewertet.

Der Erhebungsschwerpunkt dieser Arbeit ist das Bundesland Rheinland-Pfalz, da durch Experteninterviews mit den Regionalverbänden eine grobe Eingrenzung der Teilablieferung möglich war (Hein 2016; Staub 2016). Dies begründet sich darin, dass hier der größte 
Teil der Teilablieferung in Winzergenossenschaften in Deutschland stattfindet (Staub, 2016). Auch befinden sich die Winzergenossenschaften in einem wettbewerbsfähigen Umfeld mit vielen Kellereien, Erzeugergemeinschaften oder größere private Weingüter, die auch auf dem Markt für Trauben, Most und Fasswein aktiv sind. Dies ist eine grundlegende Rahmenbedingung für die Teilablieferung. Ohne einen ausreichend ausgeprägten Markt haben Genossenschaftsmitglieder keine oder nur sehr begrenzte Alternativen zur Ablieferung in einer Winzergenossenschaft, wodurch die Teilablieferung enorm an Attraktivität verliert. Durch das Marktumfeld ist zu erwarten, dass sich die teilnehmenden Genossenschaften und Mitglieder aufgrund ihrer geografischen Herkunft zwangsläufig im Alltag mit der Thematik befassen. Es wurden vier Geschäftsführer und zwei geschäftsführende Vorstandsmitglieder von sechs Genossenschaften befragt von denen drei in ihrer Genossenschaft die Teilablieferung akzeptieren und drei nicht. Insgesamt wurden sechs Genossenschaftsmitglieder befragt von denen vier Vollablieferer sind (einer war früher Teilablieferer) und zwei nutzen das Konzept der Teilablieferung. Weiterhin wurden acht Experten - Mitarbeiter von Verbänden oder andere Branchenkenner - welche nicht nur aus Rheinland-Pfalz stammen, inkludiert. Die Experten waren Vertreter involvierter Verbände, von Erzeugergemeinschaften, Kellereien und weiteren Experten, die die Branche und deren Besonderheiten seit Jahren kennen. Ihr Vorteil ist es, dass sie zum Teil als Außenstehende die Teilablieferung beobachten und deshalb das Vorgehen und die Auswirkungen erleben.

\section{Ergebnisse der experteninterviews und diskussion der Annahmen}

Im Folgenden werden die Ergebnisse aus den Experteninterviews zusammengefasst dargelegt und die jeweils dazugehörigen Annahmen praxisnah diskutiert.

Annahme 1: Teilablieferer können u. U. Rebflächen innerhalb ihres Betriebes austauschen, um Vorteile für den eigenen Betrieb zu schaffen. Dies kann sich negativ auf die Qualitätsproblematik auswirken und die Schwankungen in den angelieferten Traubenmengen erhöhen. Eine Kontrolle der Genossenschaft ist schwierig.

In der Praxis gibt es zwei legale Möglichkeiten die Vollablieferungspflicht $\mathrm{zu}$ umgehen, insofern diese nicht ohnehin von der Genossenschaft zugelassen ist: Eine Möglichkeit zur legalen Umgehung der Vollablieferungspflicht stellt die Teilung bzw. Splittung der Mitgliedsbetriebe dar, die vielen der Befragten bekannt ist und in Deutschland auch oft Anwendung findet (EXP 1-3, 5, 6, 8; GM 1-5; WG 1-5). Hierfür teilen die Mitgliedsbetriebe ihre Rebflächen auf und melden auf eine weitere Person, z.B. die Ehefrau, das Kind oder andere, einen neuen Betrieb mit einer neuen Betriebsnummer an. Der Betrieb mit der ursprünglichen Betriebsnummer bleibt vollablieferndes Mitglied einer WG, jedoch mit weniger eingebrachten Rebflächen. In den meisten Fällen werden die Rebflächen geteilter Betriebe weiter als einheitliche Gesamtfläche bewirtschaftet. Demnach ist anzunehmen, dass alle Rebflächen eines Teilablieferers ein ähnliches
Qualitätsniveau erreichen werden, auch wenn sie über unterschiedliche Vermarktungswege abgesetzt werden.

Der neu gegründete Betrieb kann völlig frei einer alternativen Vermarktung der restlichen Flächen nachgehen. Beispielsweise kann der zweite, "neue Betrieb" Mitglied in einer weiteren WG oder in einer Erzeugergemeinschaft werden (GM 3). Dies ermöglicht, dass die Rebflächen des zweiten Betriebs ebenfalls im Genossenschaftssektor verbleiben können und nicht zwangsläufig an andere Marktteilnehmer verkauft werden muss. Im Gegensatz dazu werden Rebflächen und Trauben an andere Marktteilnehmer verloren, da bei Genossenschaften mit offiziell-zugelassener Teilablieferung die Mustersatzung des DRV durch $\S 12$ den Verkauf von Trauben an eine andere WG, eine Erzeugergemeinschaft oder an ein ähnliches Unternehmen $\mathrm{zu}$ liefern verbietet (Drv 2014: §12). Hierdurch wird die Konkurrenzposition von alternativen Abnehmern, wie z.B. Weingüter oder Kellereien gestärkt und ein Rebflächenverlust des Genossenschaftssektors in Kauf genommen (Hanf \& Härle, 2015).

Ein weiterer zweiter Weg die Vollablieferungspflicht zu umgehen sind Pachtbewirtschaftungsverträge. Hierbei gibt ein Betrieb durch Pacht einen Teil seiner Flächen an andere Marktteilnehmer ab. Die WG kann dieses Vorgehen nicht verbieten und nicht dagegen vorgehen, da das Ablieferungsverfahren in deutschen WGs flächengebunden ist. Die eingebrachten Flächen sind "parzellenscharf" über die Flurstücksnummer definiert und ausschließlich diese dürfen der WG angeliefert werden. Die WG übernimmt die jeweiligen Teile der Weinbaukartei der Mitgliedsbetriebe und im Gegenzug müssen für die eingebrachten Teilflächen anteilig Geschäftsanteile der WG gezeichnet werden und bei Austritt die gleichen Kündigungsfristen eingehalten werden (EXP4; GM 4-6; WG 2, 3, 6). Die genossenschaftlichen Unternehmen betonen dabei, dass durch die offizielle Teilung der Betriebe oder durch Pachtbewirtschaftungsverträge eine ausreichende und steuerbare Trennung der Rebflächen vorhanden ist und ein Flächenaustausch ausgeschlossen werden kann.

Das früher praktizierte Teilablieferungsverfahren war deutlich freier und flexibler für die Mitgliedsbetriebe, da diese nicht an fest definierte Flächen gebunden waren, wodurch eine genaue und ausreichende Kontrolle seitens der Genossenschaft verhinderte wurde. Dies war einer der Gründe, warum das Verfahren für einige WGs zu wirtschaftlichen Schwierigkeiten führte, da die Mitglieder durch die fehlenden Kontrollen stark zu opportunistischem Verhalten tendierten und ihre angelieferten Trauben advers selektierten. Seit der Einführung der Weinbaukartei und der parzellenscharfen Mitgliedschaft ist die Kontrollierbarkeit seitens der WGs um ein Vielfaches größer geworden. Ausgereifte Kontrollmechanismen und - systeme sorgen mittlerweile für einen erhöhten Kontrolldruck seitens der Unternehmen (EXP 1, 5, 6, 8; GM 3, 5; WG 4, 6). Mitglieder wissen, dass sie häufiger und genauer kontrolliert werden und verhalten sich dementsprechend. Ein gravierender Austausch von Flächen und Mengen kann durch die neuen Kontrollmöglichkeiten weitestgehend verhindert, oder zumindest aufgedeckt und sanktioniert werden. Qualitätsmanagement-Systeme (QMS) in verschiedenen Ausführungen bieten den WGs die Möglichkeit, die Rebflächen der Mitgliedsbetriebe bereits während der 
Vegetationsphase zu kontrollieren. Sie kann somit schon früh Einfluss auf Mengen und Qualitäten nehmen. Durch dieses neu gewonnene Wissen, welche Fläche welche Erträge und Qualitäten hervorbringen wird, ist die Kontrollierbarkeit der Flächen enorm gestiegen. Auch die Überprüfung der angelieferten Durchschnittserträge hilft dabei, Fehlverhalten von teilabliefernden Mitgliedern aufzudecken. Stimmen die Durchschnittserträge eines Mitgliedes nicht mit denen der restlichen Mitglieder überein, kann die Genossenschaft gezielt Nachforschungen veranlassen. Somit können Mengen- oder Qualitätsschwankungen durchaus effektiv verhindert werden (GM 3, 4; WG 6). Ein Austausch durch Teilablieferer kann somit i. d. R. direkt bei der Anlieferung bemerkt werden und ist dadurch für den Teilablieferer nur noch sehr schwer möglich (EXP 6, 8; GM 3, 5). Durch diese neue Technologie ist es der Genossenschaft möglich, jede Weinbergsfläche jederzeit begehen zu können, auch ohne dass das betreffende Mitglied vor Ort ist (WG 4). Das Wissen, dass eine Kontrolle oder Überprüfung jederzeit, auch ohne das Beisein des Mitglieds stattfinden kann, erhöht die Hemmschwelle für Fehlverhalten enorm (WG 4, 6). Zusätzlich spielt der Faktor der sozialen Kontrolle, der in WGs unabhängig von Teil- und Vollablieferung besteht auch für die Kontrollierbarkeit von Teilablieferern einen entscheidenden Aspekt (EXP 2, 6; GM 3; WG 1). Die oftmals sehr aufmerksamen Weinbergsnachbarn verfolgen die Arbeiten der anderen Mitglieder sehr genau. Ein teilablieferndes Mitglied, das seine Flächen versucht auszutauschen, läuft Gefahr, dass der Nachbar den Austausch bemerkt und meldet.

Dies zeigt, dass Teilablieferung nicht zwangsläufig zu angelieferten Minderqualitäten oder Mengenschwankungen für Genossenschaften führen muss. Dies kann durch die Kontrollen und die Androhung von Kontrollen weitestehend eingedämmt werden. Trotz aller Kontrollmöglichkeiten kann jedoch keine lückenlose Kontrolle in der Praxis umgesetzt werden, da diese zu zeit- und kostenaufwändig ist, weshalb ein Austausch der Flächen nie vollkommen ausgeschlossen werden kann. Ein Restrisiko bleibt bestehen, dass sich Teilablieferer durch ihre Zweigleisigkeit einen Vorteil für den eigenen Betrieb verschaffen wie bspw. besteht die Möglichkeit, dass Teilablieferer die Genossenschaft als sichere Abnehmerin für unbeliebte und schwer vermarktbare Rebsorten oder schlechtere Qualitäten nutzen.

Die potentielle Gefahr, dass die angelieferten Mengen von Teilablieferern schwanken können, sehen heute vor allem sonstige Branchenexperten und Vertreter der WGs (EXP 2-4; WG 1, 2, 5). Die Schwankungen sind vorstellbar, da ein Teilablieferer mit seinen übrigen Flächen meist am freien Markt agiert und somit volatilen Preisen ausgesetzt ist. Die möglichen Schwankungen sind jedoch nach Ansicht von EXP 3, EXP 4, EXP 5 und GM 2 davon abhängig, welchen alternativen Vermarktungsweg ein Teilablieferer verfolgt. Falls eine Flaschenweinvermarktung die Alternative ist, so ist anzunehmen, dass ein Teilablieferer zuerst seine eigenen Kunden bedienen wird, bevor er Trauben an die WG liefert. In kleinen Jahrgängen kann dies bedeuten, dass die WG nur kleine Mengen erhält, da der Teilablieferer seine Selbstvermarktung voll bedienen möchte und daher zum Teil den Aufwuchs der genossenschaftlichen Flächen zusätzlich selbst verarbeiten wird. Diese Einschätzung einer potentiellen Gefahr für
Mengenschwankungen teilen jedoch nicht alle Befragten. Viele - insbesondere die Mitglieder - sehen keine vermehrten Mengenschwankungen zwischen Voll- und Teilablieferern. Mitglieder beider Ablieferungsverfahren sind lediglich naturbedingten Ertragsschwankungen unterworfen und verhalten sich der WG gegenüber vollkommen liefertreu (EXP 6, 7; GM 1-6; WG 2, 4, 6). Diese gegensätzlichen Auffassungen von Mitgliedern und Verbandsexperten bzw. Geschäftsführern zeigen verschiedene Sichtweisen auf die Teilablieferung. Insbesondere Experten aus Verbänden und Genossenschaftsführer sehen eine starke Gefährdung der Genossenschaft vertreten eine negativ geprägte Einstellung zu Teilablieferung. Im Gegensatz dazu stehen die meisten Mitgliedsbetriebe (die Teilablieferung betreiben), der Teilablieferung eher positiv gegenüber und sehen ihre Mitgliedswirtschaft dadurch besser gefördert. Sie sehen wenige bis keine Gefahren für die Genossenschaften.

Zusammengefasst kann Annahme 1 nur teilweise bestätigt werden. Das Risiko für die Genossenschaft ist vorhanden, aber eher gering und stark vom implementierten QM-System abhängig.

Annahme 2: Teilablieferer sehen die Ziele einer Mitgliedschaft in einer Winzergenossenschaft nicht alleine in den Auszahlungsleistungen, sondern auch in weiteren Faktoren, wie z. B. der Flexibilität in ihrer eigenen Betriebsausrichtung und im Flächenwachstum.

Die Hauptziele der Mitgliedschaft in einer WG von Voll- und Teilablieferern unterscheiden sich im Grundsatz nach Auffassung der Mehrheit der Befragten nicht, da beide Mitgliedsarten i. d. R. auf das Einkommen, das durch die Mitgliedschaft erwirtschaftet wird, angewiesen sind (EXP 2-6; GM 1-6; WG 1-4, 6). Das Ziel eines jeden Mitglieds ist es eine möglichst hohe Auszahlung je Hektar Rebfläche zu generieren. Das erwarten sowohl Vollablieferer, die ausschließlich auf die Leistungen der Genossenschaft angewiesen sind, als auch Teilablieferer, die lediglich einen Teil ihres Einkommens durch die WG generieren. Eine Unterscheidung ist nur dann zu erwarten, wenn Mitgliedsbetriebe den Weinbau haupt- oder nebenerwerblich betreiben, da somit eine andere Existenzgrundlage gegeben ist (EXP 6). Es ist für einige jedoch vorstellbar, dass Teilablieferer durch ihre betriebliche Zweigleisigkeit in Teilen andere Zielsetzungen verfolgen, die meist in Abhängigkeit des alternativen Vermarktungsweges $\mathrm{zu}$ sehen sind (EXP 2, 3, 6; GM 1, 5, 6; WG 5). Generell versuchen Teilablieferer den Deckungsbeitrag des größeren Betriebszweiges - den meist der alternative Vermarktungsweg darstellt $-\mathrm{zu}$ maximieren. Die restlichen Mengen, die dieser Vertriebsweg nicht vermarkten kann, liefert er an die WG, da diese eine Abnahmeverpflichtung inne hat. Gerade Teilablieferer mit Flaschenweinvermarktung werden die Ziele ihrer Selbstvermarktung fokussieren und die Mitgliedschaft in der WG mit dem Ziel einer sicheren Abnahme der übrigen Flächen sehen. Für andere ist es wiederum vorstellbar, dass das Ziel der Teilablieferer eine Vermarktung der weniger nachgefragten Rebsorten ist, bzw. die Vermarktung derjenigen Flächen, die sie selbst nicht anderweitig am Markt absetzen können (EXP 1, 2, 5, 7; GM 4, 6; WG 1). Dementgegen steht, dass teilabliefernde Betriebe wahrscheinlich ein 
hohes Qualitätsbewusstsein sowie ein großes Marktwissen besitzen, welches durch die Partizipation am freien Markt entsteht. Da Teilablieferer marktorientiert arbeiten, ist anzunehmen, dass sie versuchen werden Rebsorten und Qualitäten zu produzieren, die am Markt gefragt sind, um wirtschaftlich so erfolgreich wie möglich zu agieren. Es ist demnach eher unwahrscheinlich, dass die WG als eine Option zur risikoarmen Vermarktung von "Restmengen oder Rest-Rebsorten" gesehen wird.

Vollablieferer, die sich als Lieferant für das "eigenständige Unternehmen" Genossenschaft sehen, sind stark auf die Auszahlungen fokussiert. Teilablieferer hingegen sehen die Genossenschaft als "verlängerten Arm ihrer Mitgliedswirtschaften", die neben den Auszahlungen weitere Faktoren zur Entwicklung und Stabilisierung der Mitgliedswirtschaft dienen. So sind die Risikostreuung und die Flexibilität im eigenen Betrieb überaus wichtige Aspekte, die für Teilablieferer einen Nutzen darstellen. Durch die Möglichkeit der Mitglieder ihr unternehmerisches Risiko $\mathrm{zu}$ streuen und flexibel $\mathrm{zu}$ bleiben, bleibt die Genossenschaft attraktiv, da sie bestehende Mitglieder "emanzipiert", die eine Veränderung der eigenen Unternehmensstrukturen bevorzugen. Wenn bspw. junge Betriebsleiter erste Schritte in die eigene Vermarktung gehen möchten oder Teilmengen der Trauben über andere Kanäle abgesetzt werden. Ebenso kann die Teilablieferung interessant sein für neue Mitglieder, die bisher ihre Trauben, Most oder Wein auf dem freien Markt verkauft haben. Durch die gestiegene Konkurrenz am Markt kann der Eintritt mit Teilflächen in die Genossenschaft das Vermarktungsrisiko senken, da die Betriebe nicht mehr nur in einem Marktsegment aktiv sind. Somit könnte die Genossenschaft durch die Zulassung von Teilablieferung bestehende Mitglieder weiterhin an sich binden und neue Mitglieder anwerben. Denkbar ist aber auch, dass auf lange Sicht Mitglieder aussteigen oder Mitgliedsflächen verlorengehen, da sie durch die Teilablieferung andere wirtschaftliche Standbeine erfolgreich aufgebaut haben und diese intensivieren möchten.

Zusammenfassend kann Annahme 2 bestätigt werden. Teilablieferer sehen weitere Vorteile in der Mitgliedschaft in einer Genossenschaft wie z.B. die Chance zur Risikostreuung, mehr Flexibilität und Selbstverwirklichung.

Annahme 3: Die strategischen Betriebsausrichtungen von Voll- und Teilablieferern unterscheiden sich. Teilablieferer legen den Fokus auf ihren eigenen Betrieb, während Vollablieferer sich stärker auf die Winzergenossenschaft konzentrieren.

Hinsichtlich der Funktionen einer WG haben die Befragten sehr unterschiedliche Ansichten. So sind alle Mitgliedsbetriebe und alle WGs mit Teilablieferung der Meinung, dass Teil- und Vollablieferer keine unterschiedlichen Ansichten der Funktion einer WG haben (GM 1-6; WG 2, 4, 6). Beide Mitgliedsarten sehen in ihr ein Unternehmen, dass die Verarbeitung und Vermarktung ihrer produzierten und angelieferten Trauben übernehmen soll. Dabei haben auch Teilablieferer ein Interesse am Erfolg der WG und liefern daher oft ein breites Rebsortenspektrum an.
Im Gegenteil dazu sehen fünf der sonstigen Branchenexperten, dass die Teilablieferung durchaus einen Einfluss auf die Ansicht der Funktion einer WG nimmt (EXP 1-4, 8). Alle Teil- und Vollablieferer können sich hinsichtlich ihrer strategischen Betriebsausrichtung unterscheiden. Jeder Betrieb wird seine Ausrichtung prinzipiell am wirtschaftlich bedeutendsten Betriebszweig ausrichten. Bei Teilablieferern ist dies oftmals der alternative Vermarktungsweg, weshalb die Experten davon ausgehen, dass z.B. ein Teilablieferer mit eigener Flaschenweinvermarktung weniger den solidarischen Grundgedanken einer Genossenschaft aufweist, sondern eher eine alternative Vermarktungsoption für die Mengen sucht, die er über seine eigene Vermarktung nicht vertreiben kann. Die WG könnte in ihren Augen folglich eine Option sein, um "Restmengen" oder wenig gefragte Rebsorten eines Flaschenweinbetriebes zu vermarkten. Diese Ansicht könnte mittelfristig für WGs wirtschaftlich problematisch werden, wie Beispiele aus der Vergangenheit vermuten lassen. Die Art der alternativen Vermarktung ist hierbei ein ganz entscheidender Aspekt, weil bspw. ein Betrieb mit eigener Flaschenweinvermarktung strategisch andere Entscheidungen treffen wird, als ein Fassweinproduzent. Somit sehen Teilablieferer ein stärkeres Interesse im eigenen Betrieb und vertreten stärker die Sichtweise des "verlängerten Arms der Mitgliedsbetriebe", bei der die WG dabei hilft, die einzelnen Mitgliedswirtschaften individuell und optimal zu fördern.

Prinzipielle Unterschiede zwischen Teil- und Vollablieferer sind zum einen in der strategischen Betriebsausrichtung $\mathrm{zu}$ sehen und zum anderen in der Art der Erwerbsform (Neben- oder Haupterwerb. Vollablieferer verfolgen eine Strategie der Spezialisierung und fokussieren sich gänzlich auf die Traubenproduktion, wohingegen Teilablieferer die Diversifikation wählen, um ihr unternehmerisches Risiko zu streuen. Vollablieferer sind komplett an die WG gebunden, wohingegen Teilablieferer versuchen sich durch ihre Ausrichtung weniger von einem Vermarktungsweg abhängig zu machen, indem sie weitere Vermarktungswege neben dem der WG bedienen. Früher haben viele Betriebe diesen Effekt noch über einen landwirtschaftlichen Gemischtbetrieb erreicht. Neben Weinbau wurde z. B. noch Acker- und Obstbau oder Viehzucht betrieben und das Einkommen somit in unterschiedlichen Märkten generiert und das Risiko gestreut. Teilablieferung eröffnet Betrieben, die nicht auf eine Spezialisierungsstrategie setzen, die Möglichkeit einen Genossenschaftseintritt zu erwägen ohne Vollablieferung auszuschließen. Die potenzielle "Zielgruppe" für neue Mitglieder wird somit vergrößert.

Unabhängig ob Teil- oder Vollablieferung beide Mitgliedsarten versuchen durch eine Wachstumsstrategie konkurrenzfähig zu bleiben. Durch den voranschreitenden Strukturwandel bewirtschaften immer weniger Betriebe die vorhandenen Rebflächen, wodurch die Fläche der einzelnen Betriebe wächst.

Zusammenfassend kann Annahme 3 ebenfalls bestätigt werden. Es gibt grundlegende Unterschiede in der Strategieausrichtung. Vollablieferer sehen sich eher in einer Lieferant-Kunde-Beziehung zur Genossenschaft. Teilablieferer hingegen, sehen die Genossenschaft als 
Unternehmen, dass Möglichkeiten schafft die eigene Mitgliedswirtschaft individuell optimal zu fördern. Nichtdestotrotz gibt es aber auch Gemeinsamkeiten in der Strategieausrichtung da unabhängig ob Voll - oder Teilablieferung die Betriebe meistens ein Betriebswachstum anstreben.

\section{Zusammenfassung}

Die Ergebnisse der Studie haben gezeigt, dass die Geschäftsführer von WGs sowie die Verbandsvertreter die Vollablieferung favorisieren. Diese negative Einstellung gegenüber Teilablieferung spiegelt sich auch in der Mustersatzung für WGs, welche vom DRV bereitgestellt wird und die Vollablieferungspflicht vorgibt, wider (DRV, 2014). Dennoch bestimmt jede WG in der Praxis selbst darüber, ob die Teilablieferung zugelassen wird oder nicht - bisher haben nur wenige Genossenschaft die Teilablieferung implementiert. Die Ergebnisse belegen, dass bei den Mitgliedern ein großer Bedarf nach einem flexibleren Ablieferungsverfahren besteht und die Mitglieder in der Praxis Teilablieferung offiziell und "inoffiziell" praktizieren. Bei der "inoffiziellen" Teilablieferung umgehen die Mitglieder die Vollablieferungspflicht durch die Teilung oder Splittung der Betriebe auf zwei unterschiedliche Betriebsnummern oder die Rebflächen werden über Pachtbewirtschaftungsverträge der Genossenschaft vorenthalten. Auf diesem Wege kann die Trauben- oder Weinmenge durch Mitgliedschaften in anderen Genossenschaften im Genossenschaftswesen gehalten werden. Wahrscheinlicher ist aber, dass diese Mengen an andere Vermarktungskanäle verloren gehen. Im Gegenteil dazu verbietet die offizielle Teilablieferung durch die Mustersatzung des DRV durch $\S 12$ nichtgenossenschaftlichen Trauben der übrigen Rebflächen an eine andere WG, eine Erzeugergemeinschaft oder an ein ähnliches Unternehmen zu liefern (DRV 2014: §12). Diese stärkt somit die Konkurrenzposition von alternativen Abnehmern, wie z.B. private Weingüter oder Kellereien.

Herausforderungen in Bezug auf die angelieferten Traubenqualitäten und -menge können, aber müssen sich nicht zwangsläufig ergeben. Die Qualitäten und Mengen können durch die Kontrolle der Mitglieder / der WG in verschiedenen Formen stark aber nicht gänzlich gemindert werden. Diese Befürchtungen werden hauptsächlich von Geschäftsführern und Verbandsvertreter geteilt. Mitgliedsbetriebe sehen kaum Probleme.

Die verschiedenen Ablieferungskonzepte wirken sich auf die strategische Ausrichtung der Mitgliedsbetriebe aus, aber letztlich werden ähnliche Ziele insbesondere in Bezug auf die Höhe der Auszahlungen und des Wachstums verfolgt. Vielmehr ermöglicht die Teilablieferung eine Risikostreuung und mehr Flexibilität der Betriebsausrichtung von neuen wie auch bereits bestehenden Mitgliedsbetrieben, wodurch einem weiteren Flächen- und Mitgliederrückgang der Genossenschaften entgegengewirkt werden kann. Teilablieferung ist eine Möglichkeit bestehende Mitglieder zu "emanzipieren" und weiterzuentwickeln, um ihre Mitgliedswirtschaften individuell optimal fördern $\mathrm{zu}$ können. Gleichzeitig ist denkbar, dass durch die Teilablieferung mehr Betriebe bereit sind mit Teilflächen einzutreten, um ihre Abhängigkeit vom Vermarktungsweg sowie ihr unternehmerisches Risiko zu senken.

\section{Literatur}

J. Bijmann, Cooperatives and heterogeneous membership: Eight propositions for improving organizational efficiency. Paper presented at the EMNet-Conference, Budapest, Hungary, September 15-17 (2005)

DRV, Mustersatzung für Winzergenossenschaften, Berlin (2014)

A. Engels, Genossenschaftliche Kooperationen im Weinbau. Ein Beitrag zur Vereinbarkeit genossenschafts- und unternehmenstheoretischer Erklärungen der Kooperation, Marburg: Tectum (2002)

C.F. Altobelli, Marktforschung: Methoden - Anwendungen - Praxisbeispiele. 2. Aufl., Stuttgart: UTB Verlag (2007)

B. Frick, Does Ownership Matter? Empirical Evidence from the German Wine Industry. KYKLOS 57/3, 357 (2004)

J. Gläser, G. Laudel, Experteninterviews und qualitative Inhaltsanalyse. Als Instrumente rekonstruierender Untersuchungen. 4. Aufl., Wiesbaden: VS Verlag für Sozialwissenschaften (2010)

J.H. Hanf, M. Härle, Wirkung von Einflussstrategien auf das Verhalten von Mitgliedern am Beispiel von Winzergenossenschaften. 38th World Congress of Vine and Wine: 1-6 (2015)

J.H. Hanf, V. Belaya, E. Schweickert, Macht als Koordinationsinstrument: Überlegungen für die Agrar- und Ernährungswirtschaft,. Berichte über Landwirtschaft. 90, 429 (2012)

K.A. Hein, Leiter des Bereichs "Beratung Ware und Dienstleistung" des Genossenschaftsverband Bayern: Teilablieferung in Winzergenossenschaften innerhalb des Genossenschaftsverbandes Bayern. Telefonische Mitteilung vom 21.04.2016

P.G. Helmberger, S. Hoos, J. Farm Econ. 44, 275 (1962)

M. Heßdörfer, Geschäftsführer der Winzer der Rheinhessischen Schweiz eG: Erfahrungen und Einschätzungen zum Verfahren der Teilablieferung. Persönliche Mitteilung vom 25.04.2016

P. Jung, Betreuer/Referent des Bereichs "Weinwirtschaft" des DRV: Teilablieferung bei deutschen Winzergenossenschaften. E-Mail [Jung@drv . raiffeisen.de] vom 13.04.2016

N. Kießling, Geschäftsführer der Bezirkswinzergenossenschaft Wonnegau eG: Erfahrungen und Einschätzungen zum Verfahren der Teilablieferung. Persönliche Mitteilung vom 20.04.2016

R. Phillips, J. Farm Econ. 35, 74 (1953)

G. Schilling, Vorstandsvorsitzender der Bezirkswinzergenossenschaft Wonnegau eG: Erfahrungen und Einschätzungen zum Verfahren der Teilablieferung. Persönliche Mitteilung vom 23.04.2016

P. Staub, Betreuer/Referent der Waren- und Dienstleistungsgenossenschaften Baden- Württembergischer Genossenschaftsverband e.V.: Teilablieferung in deutschen Winzergenossenschaften. E-Mails [Patrick.Staub@bwgv-info.de] vom 18.04.2016

P.-J. Zenzen, Die Bedeutung des Weinhandels für die Entwicklung des Moselweinbaus und die Bedeutung der Winzergenossenschaften für die Entwicklung des badischen Weinbaus - Ein Vergleich. Diss (1984) 